\title{
Immunosensor development formatting for tungro disease detection using nano-gold antibody particles application
}

\begin{abstract}
The plant disease such as Rice tungro disease (RTD) becomes a major problem in rice production and also will effect in the economy loss in the country. Therefore, to tackle this problem at early stages, the immunosensor application is a most reliable sensor nowadays because of advantages towards detecting biological molecule. Thus, in order to deal with immunosensor development, it can be done by undergoing the formation of immunosensor format on screen-printed carbon electrode (SPCE). Results can be elaborated with the potential applications to detect the viruses.
\end{abstract}

Keyword: Immunosensor development; Tungro disease detection; Rice tungro disease; Tungro disease; Nano-gold antibody 